A N N A L E S Annales de Bretagne et des Pays de l'Ouest

Anjou. Maine. Poitou-Charente. Touraine

$117-3 \mid 2010$

Usages et représentations du temps dans les sociétés littorales

\title{
La gestion intégrée des zones côtières
}

Deux paradigmes pour penser le temps et l'espace : le durable et le local

Florence Gourlay

\section{(2) OpenEdition}

\section{Journals}

Édition électronique

URL : http://journals.openedition.org/abpo/1832

DOI : $10.4000 /$ abpo.1832

ISBN : 978-2-7535-1519-2

ISSN : 2108-6443

Éditeur

Presses universitaires de Rennes

\section{Édition imprimée}

Date de publication : 20 octobre 2010

Pagination : 161-169

ISBN : 978-2-7535-1276-4

ISSN : 0399-0826

Référence électronique

Florence Gourlay, "La gestion intégrée des zones côtières », Annales de Bretagne et des Pays de l'Ouest [En ligne], 117-3 | 2010, mis en ligne le 20 octobre 2012, consulté le 21 avril 2019. URL : http:// journals.openedition.org/abpo/1832 ; DOI : 10.4000/abpo.1832 


\title{
La gestion intégrée des zones côtières Deux paradigmes pour penser le temps et l'espace : le durable et le local
}

\author{
Florence GOURLAY \\ Maître de conférences en géographie \\ université de Bretagne-Sud (Lorient)
}

La gestion intégrée des zones côtières ou comment prendre en compte deux paradigmes contemporains pour penser le temps et l'espace : le durable et le local. " Nous sommes entrés dans une phase d'accélération de I'histoire, où le temps long se réduit de plus en plus aux temps courts des sociétés, voire des individus. " À cette accélération signalée par l'historien Eric Hobsbawn ${ }^{1}$ il faudrait ajouter, non pas à côté mais corrélativement et conjointement, une complexification des espaces et des échelles géographiques : des espaces imbriqués, de l'échelon local élémentaire à l'échelon le plus global qui soit, la planète.

Dans le contexte de la mondialisation, l'aménagement des territoires ne peut se concevoir sans envisager les territoires dans leur rapport au monde globalisé et sans les penser dans une perspective du long terme. Ainsi le local et le durable sont devenus en quelques années deux cadres paradigmatiques des sciences humaines. Ce que l'on nomme parfois le renouveau du local, est en fait une redéfinition de l'échelon local par nos sociétés contemporaines. Il ne s'agit pas d'un retour au localisme et aux localités, mais d'une prise en compte de la complexité du monde par les territoires et leurs acteurs.

Le temps du durable pour sa part s'exprime tout particulièrement dans le concept du développement durable. Ce concept s'est imposé dans les approches d'aménagement, à tel point qu'il constitue aujourd'hui, à travers la charte de l'environnement, un des piliers de la Constitution de la Ve République. Il oblige, mais les acceptions et les positionnements sont tellement variables, à prendre en compte l'articulation entre le court et le long terme, en posant la question de la pérennité des pratiques économiques,

1. HoBSBAWM, Eric, Entretien dans Sciences humaines, juin 2006. 
sociales, environnementales contemporaines. Cette nouvelle approche à plusieurs échelles du temps et de l'espace est un élément majeur de complexité.

Par ailleurs la complexité du monde contemporain se lit à travers d'apparentes contradictions : Immédiateté/durabilité, local/global, uniformisation/différenciation sont des exemples contemporains de ce qu'Édgar Morin appelle des antagonismes complémentaires. Un antagonisme complémentaire est l'expression du principe dialogique contenu dans la pensée complexe. Il consiste à associer deux termes, deux réalités qui, bien qu'opposées, procèdent d'une même dynamique. Ainsi sommes-nous saisi aujourd'hui par l'impérieuse nécessité de prendre en compte et de penser les antagonismes complémentaires auxquels sont confrontés de nombreux territoires, parmi lesquels le littoral. Notre questionnement sera donc le suivant : comment intégrer, dans l'action des acteurs du territoire, l'articulation entre les échelons locaux et le niveau global? Comment penser la complexité de l'exigence de durabilité (terme sur lequel il faudra s'interroger) et le temps de l'instant - la tyrannie de l'instant pour reprendre l'expression de Paul Virilio - à laquelle est soumise notre société? Comment les sociétés locales et leurs acteurs composent-ils avec ces dynamiques antagonistes mais complémentaires?

Les habitants et les acteurs des sociétés littorales doivent inventer des politiques de territoires qui permettent de prendre en compte conjointement le temps et l'espace, le durable et le local : la durabilité d'un modèle d'aménagement sur un territoire singulier. Les bouleversements climatiques et leurs conséquences sur le niveau marin sont l'exemple d'une problématique qui se définit, se réfléchit à l'échelle globale mais dont les actions liées se réalisent à l'échelle locale et du temps quotidien, c'est-à-dire à l'échelle de chaque territoire, de chaque construction socio-spatiale qui singularise les espaces littoraux. La démarche gestion intégrée des zones côtières (GIZC) est un exemple parmi d'autres d'un outil et d'un projet qui illustre cela.

Dans la présente communication nous nous intéresserons tout d'abord à la recomposition des rapports au local et au global, à l'instantané et au durable dans la société contemporaine, avant d'en illustrer un aspect en matière d'aménagement de l'espace littoral : la démarche de gestion intégrée des zones côtières en en montrant d'une part les principes et d'autre part le fossé entre ses exigences et les réalités.

La mondialisation, expression d'antagonismes complémentaires

Local/global : La recomposition des territoires

L'un des éléments structurant la société contemporaine est la redéfinition des échelles et des lieux dans un système-monde ${ }^{2}$. La mondialisation

2. Dollfuss, Olivier, "Le système-monde », dans Les Mondes nouveaux, Géographie universelle, Paris, Belin-Reclus, 1990. 
a fait émerger une échelle indépassable, le monde. À l'intérieur de cette globalité finie, chaque lieu, chaque territoire est identifié par rapport aux autres et est, que ses habitants et ses acteurs le revendiquent ou non, en relation avec le reste des territoires. Le local n'est pas la forme élémentaire de la globalité du monde. La somme des territoires n'est pas équivalente à l'échelon supérieur global. Plus que cela la complexité du monde est contenue dans chaque échelon local.

Pourtant le retour au local pourrait être perçu comme une vision passéiste du territoire, comme la tentation du repli isolationniste. Or ce renouveau du local ne se pense que par rapport au système-monde. Ainsi l'analyse du fait local ne consiste plus à effectuer une addition de connaissances sur des localités comme le faisaient certaines études de géographie d'antan, mais bien davantage à penser l'échelon local, le territoire, dans sa relation aux autres échelles, à la fois comme élément et comme totalité du monde.

Réévaluer l'échelon local (et il faudrait d'ailleurs s'interroger sur l'échelle plurielle du local : parle-t-on du quartier, de la ville, de la région? le local est tout à la fois ces différentes réalités) cela revient à penser l'inscription de chaque territoire, de chaque "local » au monde. Dans le même temps le local est aujourd'hui une des échelles qui permet l'émergence et la mise en œuvre de projet intégrant la notion de durabilité.

\section{Durable et instantané}

En même temps que se réalise une recomposition des territoires, une complexification des temps s'opère entre le durable et l'instantané. La relation nouvelle et recomposée entre local et global résonne ainsi avec un autre antagonisme complémentaire contemporain : le durable et l'instantané.

La notion de durabilité qui est inscrite dans la traduction française du concept de sustainable developement pose question. La traduction d'un développement soutenable par un développement durable fait intervenir plus directement des considérations de temps. Dès lors, le développement s'inscrit dans une dimension intergénérationnelle. Cependant l'ambiguïté naît du fait que le caractère durable d'un aménagement peut être dénué de toute éthique contenue dans le développement soutenable : les blockhaus sur les littoraux sont durables.

La notion de durabilité traduit aussi l'intériorisation croissante de la notion de risques dans nos sociétés : risque naturel, industriel, technoscientifique... Ainsi penser le risque induit un nouveau rapport au temps du court, moyen et long terme. Comme le rappelle Jean-Pierre Dupuy : « Les possibles à venir n'existent pas encore. Rétrospectivement lorsqu'ils existeront on pourra dire qu'ils étaient possibles mais ils ne le sont pas actuellement $^{3}$.»

3. Dupuy, Jean-Pierre, Pour un Catastrophisme éclairé : quand l'impossible est certain, Paris, Seuil, 2004, 214 p. 
Le paradoxe de la durabilité est que l'époque contemporaine ne cesse de surévaluer la vitesse, la rapidité, l'instantanéité, la simultanéité. Le " toujours plus vite » s'exprime à travers l'instantanéité de la communication, des transactions économiques, de la généralisation des téléphones portables qui, en permettant aux individus d'être joint partout à tout moment, "désectorise " les temps de vie et les espaces de chacun. Par cette accélération du temps, le territoire s'en trouve ainsi recomposé. Ce qu'illustre de manière fort judicieuse Jean Ollivro dans son ouvrage L'homme à toute vitesse ${ }^{4}$ en évoquant le passage " de l'ère de la lenteur homogène à celle de la rapidité différenciée ». Certains auteurs, comme Cyria Emelianoff, opérent un intéressant parallèle entre durable et global, en avançant l'idée que le durable est au temps ce que le global est à l'espace : à savoir " un élargissement de notre champ de vision au-delà du court terme ${ }^{5}$ ».

Dans ces circonstances, il est impératif de considérer les relations spatio-temporelles, et de dépasser la séparation traditionnelle entre le temps et l'espace. Comme l'écrit Pascal Buléon, ces deux réalités - le temps et l'espace - sont insécables. Mais penser l'aménagement des territoires simultanément sur le temps court (le temps de vie) et sur un temps long est un formidable défi. Il suppose d'une part une démarche volontariste à contre-courant de la vitesse et de l'instant et d'autre part la révolution intellectuelle de la complexité pour réussir à appréhender les antagonismes complémentaires. En permanence le contexte social contemporain amène les individus, les acteurs du territoire, à recombiner, à réajuster les échelles de temps et d'espace. Sur un espace singulier qu'est le littoral comment les habitants et les acteurs posent-ils le problème de cette prise en compte de ces deux paradigmes contemporains : le local dans sa résonance globale et le temps du durable et du quotidien.

Sur le littoral comme ailleurs : Réaliser le défi conceptuel de la complexité pour une harmonie sociétés/nature

De façon plus générale, le littoral est confronté aujourd'hui à une question récurrente dans notre société, celle de savoir comment parvenir à réconcilier la nature et la culture. Comment concevoir un aménagement qui réintroduise de I'harmonie dans les rapports sociétés/nature. Le véritable défi conceptuel contemporain consiste donc à intégrer davantage de complexité pour comprendre et aménager les territoires : relier les approches plutôt que de les sectoriser.

Dans un récent ouvrage Philippe Descola rappelle à juste titre que la séparation entre les deux concepts de nature et de culture est une construc-

4. Oluivro, Jean, L'Homme à toutes vitesses : de la lenteur homogène à la rapidité différenciée, Rennes, PUR, 2004, 179 p.

5. Emelianoff, Cyria, "Qu'est-ce qu'une ville durable? », Problèmes politiques et sociaux, $n^{\circ}$ 933, Dossier « La ville durable. Perspectives françaises et européennes », 2007, p. 27-28. 
tion occidentale ${ }^{6}$. II existe bon nombre d'exemples de communautés qui n'opèrent pas ce clivage entre le monde des humains et des non-humains, entre la communauté et son environnement. Réintroduire de l'harmonie sur un espace local qui résonne dans un contexte global, une harmonie garante de durabilité, voilà bien l'enjeu des sociétés littorales contemporaines. Cela passe par la construction d'une nouvelle relation à la nature autant dire qu'il s'agit d'un chantier ambitieux qui se déroulera l'échelle de plusieurs générations.

Quelques signes encourageants témoignent peut-être de cette entreprise. Sur les territoires littoraux, la démarche gestion intégrée des zones côtières en est par exemple l'illustration. La GIZC est une démarche qui tend à favoriser une approche complexe de l'aménagement des territoires.

La Gestion intégrée des zones côtières :

entre procédure et projet de territoire dans l'ère planétaire

Une démarche volontariste et ambitieuse

La notion de gestion intégrée trouve ses origines dans les principes de l'Agenda 21 formulés au sommet de la Terre de Rio de 1992, réunion internationale qui a été un important vecteur et diffuseur du concept de développement durable. La gestion intégrée consiste donc à instaurer sur les littoraux une démarche concertée d'application des principes du développement durable, c'est-à-dire, comme il est défini dans le rapport Brundtland de 1987, un développement permettant de satisfaire les besoins des sociétés du présent sans compromettre les capacités des sociétés futures à satisfaire les leurs.

Une meilleure gestion des zones côtières est inscrite dans les priorités de I'Union européenne. Il est vrai que la plupart des zones côtières européennes sont soumises à des niveaux de pressions anthropiques relativement élevés. Presque $50 \%$ de la population européenne vit à moins de 50 kilomètres des côtes et les ressources économiques générées par cette frange représentent une bonne partie de la richesse de I'Union (pêche, transport, tourisme, industrie et commerce). Or l'utilisation intensive et croissante des ressources côtières a des effets sur la qualité des eaux et la disponibilité de la ressource hydrique, l'accentuation de l'évolution des traits de côtes. Par conséquent, à terme, existe un fort risque de " dévalorisation du capital naturel de ces zones ", pour reprendre la terminologie européenne, induisant aussi une dégradation des conditions socio-économiques sur ces territoires. Ce constat a conduit le Parlement européen et le Conseil à approuver le 30 mai 2002 la recommandation sur l'application de la gestion intégrée des zones côtières qui vise à encourager l'élaboration de stratégies de gestion intégrée permettant d'orienter les zones côtières vers des scénarios plus durables.

6. Descola, Philippe, Par-delà nature et culture, Paris, Gallimard, 2005, 623 p. 
En France, la prise en compte de cet axe politique a fait l'objet du conseil interministériel d'aménagement et de développement durable des territoires (CIADDT) de septembre 2004 qui a lancé la dimension opérationnelle sur l'ensemble des littoraux français par la mise en place d'une stratégie nationale du littoral. Un appel à projets est lancé pour financer des expériences GIZC sur des territoires dont les 25 projets lauréats ont été connus en juillet 2005.

\section{À la fois un outil et un projet de territoire}

L'intérêt de la GIZC est à la fois d'être une méthodologie innovante de gestion littorale mais aussi les conditions de création et d'émergence d'un contexte favorable à raisonner différemment sur le littoral. Avec cette démarche on se situe donc bel et bien dans un processus complexe de récursivité organisationnelle dans la mesure où le phénomène produit le phénomène qui le produit.

Encore faut-il que la démarche ait un contenu en termes d'actions portées et conduites par les acteurs du littoral : les élus politiques, les représentants des services de l'État (DDE, affaires maritimes, Ifremer), les pêcheurs, les agriculteurs, les résidents permanents, secondaires, les entreprises... Le premier élément de la démarche GIZC est de faire se rencontrer et discuter ensemble les différents acteurs des sociétés littorales. Cette dimension est contenue dans la notion d'intégration. II s'agit d'une notion centrale pour viser aux décloisonnements des approches, au croisement des regards.

L'intégration suggérée est d'abord verticale : il s'agit de combiner de multiples échelles territoriales de décisions de l'international au local. Elle est ensuite horizontale c'est-à-dire intersectorielle et inter-institutionnelle. On cite souvent à ce propos l'exemple de la ria d'Étel où une association milite pour une meilleure compréhension et action entre ostréiculteurs et agriculteurs, ces derniers étant responsables en partie en amont de la qualité des eaux des concessions ostréicoles. L'enjeu est de trouver les moyens de concilier et de pérenniser les deux activités.

L'intégration suggère aussi celle des différentes programmations de planification ou de réglementation territoriale qui couvrent et recouvrent de larges périmètres littoraux : loi littorale, schéma de cohérence territoriale, schéma de mise en valeur de la mer... La démarche milite donc dans le sens d'un décloisonnement des approches entre les différents acteurs des espaces et des temps du littoral. II s'agit de prendre en compte le temps des acteurs la terre et ceux de la mer, l'espace maritime/l'espace terrestre, le durable/l'instantané, l'échelon local et ses résonances globales.

La GIZC, processus récursif organisationnel, repose au départ sur un groupe porteur, sur une initiative. II faut une impulsion qui est toujours le fruit d'une situation locale particulière : une institution qui peut-être un service déconcentré de l'État (DDE), un ingénieur, un technicien d'une collec- 
tivité locale, un élu politique, une association... Dans ce contexte le choix d'inscrire un territoire dans une démarche GIZC est l'expression à un moment donné, dans un contexte donné d'une volonté politique forte. II faut donc parler aussi de projet de territoire à propos de cette démarche qui fait le pari d'ancrer un territoire dans un processus d'aménagement décloisonné, transversal, inscrit sur le temps du quotidien et du temps long.

Des exigences fortes mais des mises en place difficiles

Une obligation : décloisonner les approches,

les modes de pensée et de fonctionnement

L'intérêt majeur de la démarche GIZC est qu'elle tente de décloisonner les approches et les fonctionnements des structures de gestion du littoral en favorisant le croisement des regards entre les différents acteurs. Faire parler un ostréiculteur avec un plaisancier, un agriculteur, un élu avec un pêcheur, un universitaire... Ce décloisonnement est louable et essentiel pour appréhender la complexité des situations d'aménagement contemporaines. Cependant derrière les discours officiels affichés, la capacité réelle des acteurs à varier les approches, à combiner les temporalités et spatialités n'est pas toujours aussi évidente. D'autant plus que le temps du politique n'est pas celui du pêcheur, de l'agriculteur, du touriste, du scientifique, de l'entrepreneur. De plus et/ou par conséquent le contenu mis derrière le terme durable varie largement selon les acteurs. Certains s'accommodent fort bien d'une approche purement technicienne du développement durable ne remettant nullement en cause une pratique économique pernicieuse, d'autres voudront voir privilégiée au contraire une approche plus radicale.

\section{Des projets français assez décevants}

Sur les quarante-neuf dossiers qui ont été soumis à l'ex-DATAR (devenue depuis le $1^{\text {er }}$ janvier 2006 la direction interministérielle à l'aménagement et la compétitivité des territoires), seuls 25 ont été retenus. À la lecture des fiches rédigées en réponse à l'appel à projets "Pour un développement équilibré des territoires littoraux par une gestion intégrée des zones côtières » la prise en compte de la nécessaire transversalité et décloisonnement des approches est variable. Bien sûr la seule analyse des fiches-projets est insuffisante pour évaluer la réalité d'une action, et il faudrait pouvoir connaître chaque contexte territorial, chaque contexte politique local (au sens large du terme, types d'acteurs, jeux d'acteurs) pour pouvoir juger de la réalité de l'intégration des principes de la GIZC. Cependant le soin apporté à la rédaction des projets est révélateur de la prise de conscience de la complexité assumée de la gestion des littoraux. À ce titre les formulations lapidaires de certains projets signifient-elles une version tronquée et étriquée de la GIZC (exemple : Pays de Cornouailles) ? À l'opposé, la 
fiche-projet présentée par l'agglomération lorientaise est particulièrement détaillée et intéressante. Pour autant lorsque l'on connaît la situation politique locale, le jeu des acteurs, il est difficile de croire que ce projet de nouvelle démarche pour traiter les questions relatives aux espaces littoraux et maritimes lorientais puisse être opérationnel dans l'immédiat.

La déception à la lecture des fiches-projets est d'une certaine façon à la hauteur de l'ambition du projet général de GIZC. Sur les vingt-cinq projets retenus représentants l'ensemble des littoraux français, la référence à la transversalité apparaît souvent. Hormis quelques cas très isolés où la démarche GIZC se résume à une approche sectorielle ou par une infrastructure ${ }^{7}$, tous les projets donnent des éléments allant dans le sens d'une volonté de créer les conditions d'une intégration de la complexité dans l'appréhension des espaces littoraux. Les termes associant les notions de concertation, " action concertée », de processus partenarial, de participation, du croisement des regards, de la vision partagée par les acteurs des enjeux territoriaux sont présents dans 16 des projets.

La GIZC se veut en même temps un outil et une démarche. La demande de la part des décideurs locaux en termes de méthodologie d'application est forte et démontre assez bien la difficulté institutionnelle, politique, sociale, intellectuelle en un mot culturelle, qu'il y a à mettre en action les principes de la gestion intégrée. Or sur cet aspect les projets restent assez évasifs. La véritable question qui se pose aujourd'hui est celle du comment. Comment réaliser ce défi conceptuel auquel nous invitent toutes les analyses contemporaines qui s'attachent à monter la difficulté qui existe pour appréhender la société contemporaine? Quelles capacités nos sociétés ontelles pour mettre en pratique ces principes? Les difficultés identifiées sont liées à plusieurs éléments : difficulté de combiner les temps, le long terme, le court terme, le temps du scientifique, du mandat politique, de l'institution (fréquence des changements ministériels). Par ailleurs les démarches GIZC demeurent pour le moment cantonnées à des approches scientifiques et techniciennes, les sciences humaines sont encore trop souvent absentes des réflexions en amont des projets et dans la mise en place des actions. Cela traduit parfaitement la difficulté qui existe pour casser les clivages disciplinaires.

Pour conclure, nous avons montré que la démarche GIZC contenait un projet ambitieux de mener autrement par les acteurs des politiques de gestion du littoral. Il nous faut souhaiter, comme le suggère Jean-Paul Ferrier militant pour un contrat géographique ${ }^{8}$, que l'époque contemporaine avec

7. C'est l'exemple à la Martinique avec la réalisation d'un centre de la mer à vocation touristique, éducative et de formation sur le milieu marin en baie du Robert. Ou encore celui du projet de Monaco qui concerne exclusivement la prise en compte des risques de pollution marine.

8. Ferrier, Jean-Paul, Le Contrat géographique ou l'habitation durable des territoires, Paris, Payot, 1998, 251 p. 
la redéfinition des réalités temporelles et spatiales qu'elle produit et qui la produisent nous fasse entrer dans " un nouvel âge du faire ». En effet, dans un contexte de revalorisation des approches locales, il apparaît que les acteurs, les habitants ont les moyens de construire, de fabriquer de nouvelles réalités territoriales : reste à savoir si le défi conceptuel de la pensée complexe peut être relevé.

RÉSUMÉ

Avec la mondialisation, la recomposition des territoires s'accompagne d'une complexification de l'articulation entre l'instantané et le durable. L'article pose la question suivante : Comment, à une époque marquée, d'un côté, par l'accélération des communications, des transactions, des prises de décision, d'un autre côté par la prise en compte de la durabilité, les sociétés locales et leurs acteurs composent-ils avec ces exigences parfois contradictoires? L'étude de l'expérience de Gestion intégrée des zones côtières (GIZC) apporte des éléments de réponse. La gestion intégrée consiste à instaurer sur les littoraux une démarche concertée d'application des principes du développement durable. Mais on observe que le temps du pêcheur, ou celui de l'ostréiculteur, n'est pas celui de l'agriculteur, du touriste, de l'élu ou de l'expert. L'auteure attribue à ces décalages les ratés des politiques intégrées de gestion des zones côtières. Chacun apprécie en effet selon des critères bien spécifiques la notion de "durabilité », désormais au cœur des politiques d'aménagement.

\section{ABSTRACT}

How can local societies take into account the growing demand for durable development in a time of speeding-up of communications, transactions, decision making? How do actors involved in country planning face those conflicting constraints? To answer this question, the author studies the implementation of the Integrated management of coastal zones (Gestion intégrée des zones côtières, GIZC). The purpose of the GIZC is to encourage dialogue between different actors involved in politics of durable development of the littoral. But fishermen, oyster-farmers, farmers, tourists, experts, local politicians do not share the same conception of time and "durability", a core-concept of territorial planning. The failure of Gestion intégrée des zones côtières must be attributed to those differences. 

Erratum au $n^{\circ} 1$ du tome 117, année 2010

Le texte en bas de la page 45 (Laurence Moal, « Entre méfiance et accueil ») est tronqué ainsi que la note 28 , incomplète. En voici les compléments.

[...] le duché par des efforts importants qui se concentrent sur des sites clés, considérés comme les portes d'entrée de l'armée anglaise par lesquelles des flottes anglaises ont déjà débarqué ou sont susceptibles d'effectuer une descente. Ces places sont renforcées par des garnisons et des travaux de fortification. Brest, aux mains des Anglais depuis la guerre de succession, est récupérée par le duc en 1397 et fait l'objet d'importants travaux pour son entretien. Un château entièrement neuf est bâti à Vannes (château de l'Hermine) et celui de Nantes est agrandi. En 1419-1421, le duc, un moment [...]

28. Russon, Marc, «Trémazan et la mer au xve siècle », Le Trémazan des Du Chastel : du château fort à la ruine, Actes du colloque de Brest, 10, 11 et 12 juin 2004, sous la direction d'Yves Coativy, Association SOS Château de Trémazan, Brest-Landunvez, CRBC, 2006, p. 300. 



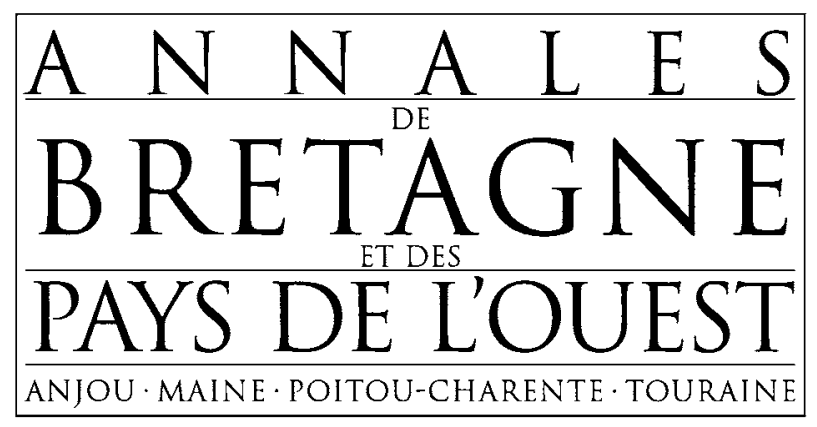

\section{Abonnement}

FRANCE, 4 numéros : $32 €$ - Étranger, 4 numéros : $40 €$ (franco de port)

Année 20...

\section{ADRESSE D'EXPÉDITION \\ (pour toute commande)}

Nom

Prénom

Organisme

Adresse

Code postal

Ville

Pays

Ce bon de commande accompagné d'un chèque à l'ordre de Agent comptable de l'Université Rennes 2 est à envoyer à l'adresse suivante:

\section{Presses Universitaires de Rennes}

Campus de La Harpe 2 rue du Doyen-Denis-Leroy

35044 Rennes cedex 


\section{QUELQUES RĖGLES DE PRÉSENTATION DES TEXTES À L'USAGE DES AUTEURS}

1 - Indiquer sous le titre de l'article la qualité et l'adresse institutionnelle de l'auteur

Ex. : Xxxx Xxxxxx, maître de conférences en histoire contemporaine, université de X., groupe de recherches de rattachement.

2 - Remplacer les majuscules par des petites capitales pour les noms d'auteurs et les siècles: $\mathrm{XV}^{\mathrm{e}}$ siècle et non $\mathrm{XV}$ siècle.

Accentuer les majuscules.

3 - Abréviations : en user le moins possible

Pas d'abréviations pour le nom des revues, surtout pour les revues locales qui ne sont connues que des locaux.

Pour les dépôts d'archives:

- ne pas mettre ADIV ou AD 35 mais : Arch. dép. d'llle-et-Vilaine, du Morbihan...;

- préférer Arch. nat. à AN;

- mettre les noms des bibliothèques en entier.

Développer les sigles entre parenthèses lors de leur première occurrence.

4 - Références bibliographiques

- mettre toujours et partout les prénoms et les noms (ne pas abréger le prénom par une initiale);

- utiliser les petites capitales pour le nom propre et non les majuscules, utiliser les minuscules pour le prénom; les séparer par des virgules.

Nom, Prénom, Titre de l'ouvrage en italique, Lieu d'édition, éditeur, «collection » (éventuellement), année, pages.

Une bibliographie peut être donnée en fin d'article si cela est nécessaire.

5 - Forme des notes

Préférer la forme traditionnelle (référence biblio. développée lors de sa première occurrence, abrégée ensuite) à la forme anglo-saxonne (nom, date, page).

6 - Titres et sous-titres

Il est préférable de s'en tenir à deux niveaux. Mais si cela s'avère nécessaire afin de ne pas obtenir de trop longues pages trop compactes, un troisième niveau d'intertitres peut être utilisé.

7 - Cartes et documents photographiques

- Les cartes au trait devront être d'une EXCELLENTE QUALITÉ GRAPHIQUE sans collage hasardeux ni légende manuscrite.

- Il est toujours préférable de FOURNIR LES ORIGINAUX, sinon, un fichier informatique au format .EPS, .Al pour les cartes et plans ou .TIF pour les photographies. en aucun cas les images seront intégrées au fichier word seulement : IL FAUT ÉGALEMENT LE FICHIER SOURCE.

8 - Autres questions non évoquées ici... consulter la rédaction à l'adresse ci-dessous.

9 - Donnez une adresse mail et téléphone où l'on puisse vous joindre facilement au moment où l'on fait la mise en page de votre article.

\section{Contact}

Annie ANTOINE, Secrétaire de l'Association pour la publication des ABPO université de Rennes 2, Département Histoire,

Place du Doyen-Henri-Le-Moal - 35043 Rennes Cedex e-mail : annie.antoine@uhb.fr

Les manuscrits sont à envoyer à l'adresse ci-dessus

Ouvrage achevé d'imprimer

sur les presses du service reprographique

de l'université Rennes 2 Haute-Bretagne

en octobre 2010

Imprimé en France 